\title{
Panretinal laser photocoagulation for proliferative diabetic retinopathy: the effect of laser wavelength on macular function
}

\author{
Chris Canning, Philip Polkinghorne, Azrin Ariffin, Zdenek Gregor
}

\begin{abstract}
The effect on macular function of the wavelength of laser light used to treat proliferative diabetic retinopathy was studied. Thirty-six eyes of 25 patients received panretinal photocoagulation according to a standardised protocol and before and at intervals after the treatment were tested by a battery of macular function tests. Each eye was randomly assigned to treatment with blue-green, yellow, or orange laser. The tests included best corrected visual acuity, colour vision, contrast sensitivity, macular threshold, and central visual fields. There were no significant differences in the results of these tests between the three treatment groups, though there was wide scattering of baseline values within each group. Laser wavelength has not been shown to be an important determinant of central visual function following panretinal photocoagulation in patients with proliferative diabetic retinopathy within the wavelengths tested.
\end{abstract}

Panretinal laser photocoagulation is an established and effective treatment for proliferative diabetic retinopathy. ${ }^{12}$ Argon blue-green lasers have been used most commonly. There is, however, increasing awareness of the potential phototoxic hazards of short wavelength lasers both for the operator ${ }^{34}$ and for the patient. ${ }^{5}$ The aim of the present study was to determine by a battery of macular function tests, whether macular phototoxicity could be demonstrated after panretinal laser photocoagulation, as currently practised, and if so whether longer wavelengths produced less of an effect than others.

\section{Patients and methods}

Patients attending Moorfields Eye Hospital with previously untreated proliferative diabetic retinopathy were invited to participate in the study. Patients were eligible if they had proliferative diabetic retinopathy, either with disc new vessels or with new vessels elsewhere, as assessed by biomicroscopy and fluorescein angiography. Their eyes had a visual acuity of $6 / 12$ or better, a normal intraocular pressure, clear media, no clinically significant macular oedema as defined by the Early Treatment Diabetic Retinopathy Study Research Group, and no other pre-existing ocular pathology. Informed consent was obtained, and the patients agreed to a protocol approved by the Ethical Committee at this institution.

Prior to treatment each patient had a full ophthalmic assessment which included slit-lamp biomicroscopy and binocular indirect ophthalmoscopy. All patients had fluorescein angiography and fundus colour photography prior to laser treatment.

At entry the study eyes were randomised to treatment with one of three wavelengths; bluegreen $(488 / 514 \mathrm{~nm})$, dye yellow $(577 \mathrm{~nm})$, or dye orange $(595 \mathrm{~nm})$. All treatments were carried out on the coherent dye laser. Where both eyes of the same patient were enrolled, one was treated with argon blue-green, and the other was randomly assigned to dye yellow or orange. Follow-up fluorescein angiography was undertaken at the end of the study period (three months) to assess macular perfusion and confirm regression of the new vessels.

The patients were treated through a maximally dilated pupil with a Rodenstock lens. The Goldmann three-mirror lens was also used on four eyes where only partial treatment could be obtained with a Rodenstock lens. The patients were treated in a single treatment session with topical anaesthesia (amethocaine). Two thousand burns were applied to each eye, of spot size $200 \mu \mathrm{m}$ and duration 0.1 second. In those eyes in which treatment was completed with a three-mirror lens, the spot size was increased to $500 \mu \mathrm{m}$. The power was adjusted to produce just noticeable immediate blanching of the retinal pigment epithelium. No additional laser treatment was given during the three-month period needed to complete the study.

The battery of macular function tests described below was done before photocoagulation and then one week, one month, and three months after treatment. All the psychophysical measurements were performed under standard conditions of illumination with the exception of the visual fields. For this investigation the patient was dark adapted for five minutes and then tested under mesopic conditions.

The best corrected visual acuity was recorded on a projected Snellen chart at a distance of 6 metres. Colour vision was assessed with a Farnsworth-Munsell 100-hue test. The error score was calculated from the sum of differences between the number of each cap and its standard position. ${ }^{7}$ Contrast sensitivity was evaluated by both the Arden grating and Vistech plates. ${ }^{89}$ Contrast sensitivities were measured between 0.2 and 6.4 cycles per degree for the Arden gratings and between 0.5 and 22.8 cycles per degree for the Vistech gratings. Macular thresholds were assessed with the Friedmann Mark 1 field analyser from four locations in the central $5^{\circ}$.

The psychophysical data from the three treat- 


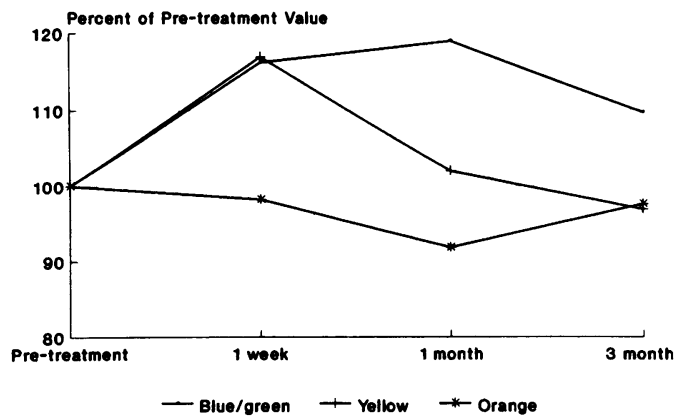

Figue $1 A$

Figure 1 Results of data from three groups of patients treated with bluelgreen (488/ $514 \mathrm{~nm})$, yellow ( $577 \mathrm{~nm})$, and orange $(595 \mathrm{~nm})$. The results are shown as the mean of the post-treatment values as a percentage of the pretreatment value for each test: (A) FarnsworthMunsell 100-hue test; $(B)$ Arden gratings; $(C)$ Vistech plates; $(D)$ Central field threshold.

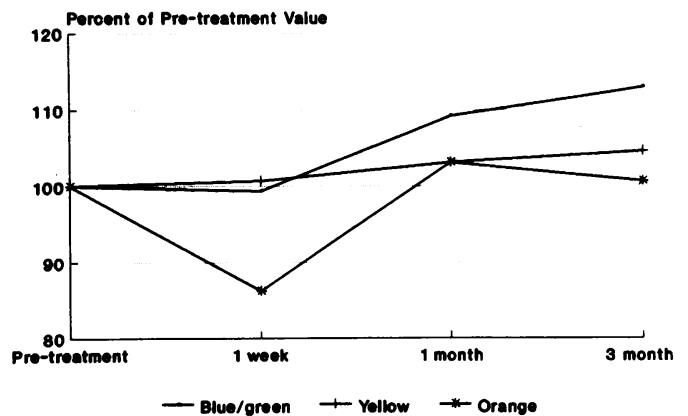

Figure 1C ment groups were compared by a masked observer. With the exception of the Snellen acuity the results were analysed according to the general linear model (GLM) procedure using a split plot design with the program SAS. The data satisfied the requirements of type $H$ covariance, so adjustment factors were neither needed nor employed. In addition separate analysis was made for each psychophysical parameter as a function of time. In this later analysis significant main and interaction effects were investigated by Tukey's HSD ('honestly significant difference'). A significance level of $5 \%$ was maintained for all analyses.

\section{Results}

Thirty-six eyes of 25 patients were included in the study. Fifteen eyes were treated with argon blue-green, 10 with dye yellow, and 11 with dye orange. The patient's characteristics in the three treatment groups were similar; their mean age was 48 years (range 20-72 years) and half were women. Sixteen of the patients were insulin dependent, the remainder being controlled with oral hypoglycaemic agents.
Table 1 Results: value(standard deviation)

\begin{tabular}{|c|c|c|c|c|}
\hline & Pretreatment & l week & 1 month & 3 months \\
\hline \multicolumn{5}{|l|}{ Farnsworth-Munsell 100-hue } \\
\hline $\begin{array}{l}\text { Blue/green } \\
\text { Yellow } \\
\text { Orange }\end{array}$ & $\begin{array}{l}223(180) \\
254(141) \\
207(140)\end{array}$ & $\begin{array}{l}251(190) \\
255(123) \\
213(154)\end{array}$ & $\begin{array}{l}240(166) \\
240(126) \\
206(154)\end{array}$ & $\begin{array}{l}231(168) \\
237(139) \\
205(151)\end{array}$ \\
\hline \multicolumn{5}{|l|}{ Arden grating } \\
\hline Yellow & $85(13)$ & $80(15)$ & $78(16)$ & $79(16)$ \\
\hline \multicolumn{5}{|l|}{ Vistech plates } \\
\hline Blue/green & $17 \cdot 8(5 \cdot 7)$ & $17 \cdot 6(5 \cdot 4)$ & $18 \cdot 6(4 \cdot 9)$ & $19 \cdot 3(5 \cdot 2)$ \\
\hline Yellow & $16.9(7.9)$ & $15 \cdot 5(5 \cdot 8)$ & $16 \cdot 3(6 \cdot 5)$ & $16 \cdot 1(5 \cdot 1)$ \\
\hline Orange & $17 \cdot 0(9 \cdot 0)$ & $16 \cdot 9(5 \cdot 2)$ & $19.9(5 \cdot 8)$ & $19 \cdot 6(5 \cdot 8)$ \\
\hline Central field threshold & $2 \cdot 2(0 \cdot 46)$ & $2 \cdot 1(0.39)$ & $2 \cdot 1(0.55)$ & $2 \cdot 2(0.46)$ \\
\hline Yellow & $2 \cdot 2(0 \cdot 62)$ & $1.9(0.71)$ & $1.9(0 \cdot 71)$ & $2 \cdot 0(0.67)$ \\
\hline Orange & $2 \cdot 3(0 \cdot 40)$ & $2 \cdot 2(0 \cdot 48)$ & $2 \cdot 4(0.42)$ & $2 \cdot 4(0 \cdot 32)$ \\
\hline
\end{tabular}

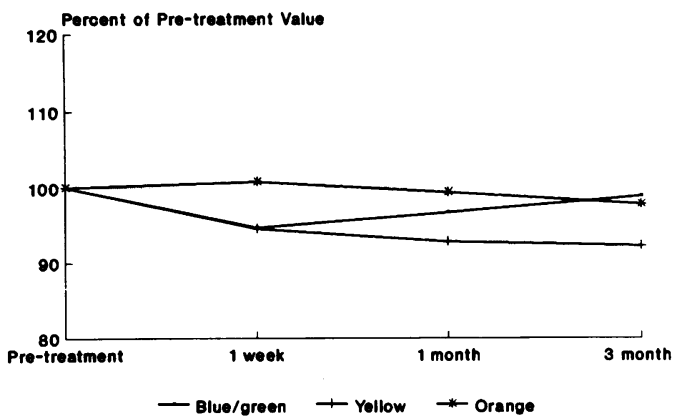

Figure 1B

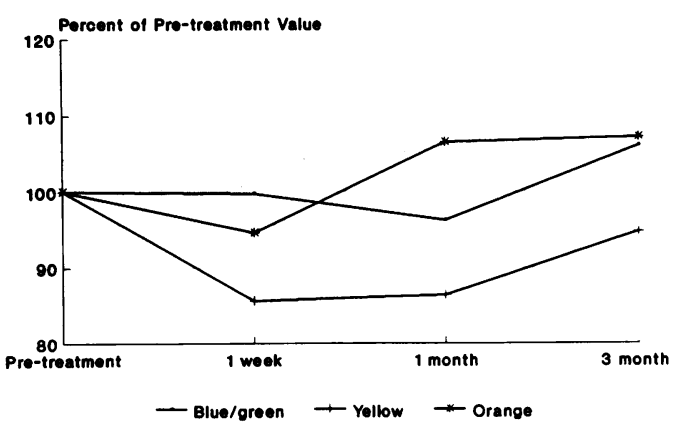

Figure 1D

The data presented graphically (Fig 1) have been normalised to show the change of posttreatment values as a percentage of their pretreatment levels. There is a trend for the Farnsworth-Munsell 100-hue value to be worse after treatment with shorter wavelength laser and to take longer to return to the baseline. Little change occurred pre- to post-treatment in the Arden plate or Vistech data, while in all groups there was a consistent reduction in central field threshold a week after treatment which later recovered. No trend was shown to be statistically significant.

\section{Discussion}

Xenon arc and heavy, long-duration-burn argon blue/green laser panretinal photocoagulation in diabetics have been shown to reduce macular function as measured by the FarnsworthMunsell 100-hue test. ${ }^{11}$ There is increasing awareness of the unique sensitivity of the retina to blue light. ${ }^{1213}$ Acute macular phototoxicity after short but not long wavelength laser exposure can be demonstrated in both the operator ${ }^{3+}$ and in patients treated for peripheral retinal tears with previously normal colour vision."

A wide variation was found in the pretreatment values for the psychophysical parameters, particularly colour vision, which made the standard deviations quite large (Table 1). This weakened the statistical comparisons between the three treatment groups.

This study was undertaken to determine whether the use of longer wavelength laser for panretinal photocoagulation could be shown to produce a significantly better macular outcome than argon blue-green laser. Current treatment protocols in panretinal photocoagulation call for short duration $(0 \cdot 1$ second or less), light (just visible grey-white spots) burns. The main observation to come out of this study is how poor and 
variable macular function is even in a group of diabetics with proliferative retinopathy selected for their good pretreatment visual acuity. The standard deviation of the baseline tests was large, and the values themselves were worse than those in non-diabetic patients. Similar changes have been noted before ${ }^{1415}$ and may have been the main factor which prevented demonstration of the more subtle phototoxic effects of shorter wavelength lasers. This may also explain the similarity of outcomes in patients treated by grid photocoagulation for diabetic macular oedema with argon blue-green and krypton red lasers. ${ }^{16}$

This study demonstrates that the diabetic eye is a poor model for the assessment of the clinical side effects of lasers, but the non-significant results as detailed above should not be taken as evidence that short wavelength lasers are as safe as longer wavelength ones. There is gathering proof in favour of longer wavelengths, and it seems advisable to avoid blue laser light wherever possible. ${ }^{3-5}$

1 Diabetic Retinopathy Study Research Group. Photocoagulation treatment of proliferative diabetic retinopathy: clinica application of diabetic retinopathy study (DRS) findings.
DRS Report number 8. Ophthalmology 1981; 88: 583-600.

2 Little HL. Proliferative retinopathy: pathogenesis and treatment. In: Little HL, Jack RL, Patz A, Forsham PH, eds Diabetic retinopathy. New York: Thieme-Stratton, 1983

3 Berninger TA, Canning CR, Gunduz K, Strong N, Arden GB
Using argon laser blue light reduces ophthalmologists' colour contrast sensitivity. Arch Ophthalmol 1989; 107 1453-8.

4 Gunduz K, Arden GB. Changes in colour contrast sensitivity associated with operating argon lasers. $\mathrm{Br} \mathcal{F}$ Ophthalmol 1989; 73: 241-6.

5 Canning CR, Strong N, Berninger TA, Arden GB. Colour contrast sensitivity changes caused by peripheral retinal laser photocoagulation. Eye in press.

6 Early Treatment Diabetic Retinopathy Study Research Group. Photocoagulation for diabetic macular edema. Early Treatment Diabetic Retinopathy Study report number 1 Arch Ophthalmol 1985; 103: 1796-806.

7 Farnsworth D. The Farnsworth Munsell 100 hue test and dichotomous tests for colour vision. F Opt Soc Am 1943; 10 568-78.

8 Arden GB, Jacobson JJ. A simple grating test for contrast sensitivity: preliminary results indicate value in screening for glaucoma. Invest Ophthalmol Vis $S$ ci 1978; 17: 23-32.

9 Ginsburg APJ. A new contrast sensitivity vision chart. Ophthalmic Physiol Opt 1984 61: 403-7.

10 Birch J, Hamilton AM. Xenon arc and argon laser photocoagulation in the treatment of diabetic disc neovascularisation. Part 2 - effect on colour vision. Trans Ophthalmol Soc UK

11 Birch J. Colour vision changes following different types and amounts of argon laser photocoagulation in the treatment of diabetic retinopathy. Doc Ophthalmol Proc Ser 1987; 46 $31-6$.

12 Ham WT Jr, Mueller HA, Sliney DH. Retinal sensitivity to damage from short wavelength light. Nature 1976; 20: 1105 11.

13 Mainster MA. Wavelength selection in macular photocoagulation: tissue optics, thermal effects and laser systems. tion: tissue optics, thermal
Ophthalmology 1986; 93: 952-8.

14 Peduzzi M, Longanesi L, Ascari A, et al. Screening of early color vision loss in diabetic patients. $\mathcal{F}$ Fr Ophtalmol 1989; 12: 791-6.

15 Frank RN. Visual fields and electroretinography following extensive photocoagulation. Arch Ophthalmol 175; 93: 591-8.

16 Casswell AG, Canning CR, Gregor ZJ. Treatment of diffuse diabetic macular oedema: a comparison between argon and krypton lasers. Eye 1990; 4: 668-72. 